\title{
Uso de tarjeta universitaria y tecnología móvil para el control de acceso a instalaciones universitarias y su posterior análisis en términos de rendimiento académico y control de asistencia en clase Use of university card and mobile technology to study and improve academic performance and class attendance control
}

\author{
Alejandro Rodríguez-González ${ }^{1,3}$, Diego Fernandez Peces-Barba ${ }^{1,3}$, Francisco Borja Lorenzo-Gomez ${ }^{1,3}$, Luis Pulido \\ Garcia-Duarte $^{3}$, Angel Garcia-Pedrero ${ }^{3}$, Ernestina Menasalvas ${ }^{1,3}$, Consuelo Gonzalo ${ }^{1,3}$, Victor Robles ${ }^{1,2,3}$, Maria Boyer \\ Lagos $^{2}$, Maria I. Domecq Urquijo ${ }^{2}$, Maria J. Carrillo Troya ${ }^{2}$, Juan L. Esteban Uceda ${ }^{2}$ \\ \{alejandro.rg, ernestina.menasalvas, consuelo.gonzalo, victor.robles, maria.boyer, mariaisabel.domecq, mariajose.carrillo, \\ juanluis.esteban \}@upm.es \\ \{ angel.garcia, luis.pulido\}@ctb.upm.es \\ \{ dfernandezp, fb.lorenzo \}@alumnos.upm.es
}

${ }^{1}$ ETS Ingenieros Informáticos, ${ }^{3}$ Centro de Tecnología Biomédica

Universidad Politécnica de Madrid Madrid, España

\author{
${ }^{2}$ Vicerrectorado de Servicios Tecnológicos \\ Universidad Politécnica de Madrid \\ Madrid, España
}

\begin{abstract}
Resumen- Hoy en día es habitual que las universidades ofrezcan a sus alumnos carnés que los identifican con el fin de poder hacer uso de las instalaciones universitarias, así como de identificarse como estudiantes fuera del ámbito universitario. De igual modo, es cada vez más frecuente que dichos carnés incorporen las últimas tecnologías, como las contactless NFC o RFID para hacer un uso más dinámico del carné. El presente artículo explora el desarrollo de un nuevo servicio a implantar en las universidades mediante el uso del carné con tecnología contactless $\mathrm{y}$, dispositivos móviles. Concretamente los objetivos del servicio son, por una parte, permitir el control de acceso de los usuarios potenciales de las bibliotecas, y por otra parte, su aplicación en las aulas de clases como medio de control de asistencia para sustituir a los procedimientos basados en firmas. Finalmente, el proyecto presenta múltiples posibles explotaciones y casos de uso adicionales que pueden ser aplicados en base a la explotación de los datos recogidos y que permitirán analizar y fomentar un mejor rendimiento académico, por parte de los estudiantes, así como una mejor gestión de las instalaciones universitarias. El caso de estudio de aplicación del proyecto descrito en el presente artículo es la Universidad Politécnica de Madrid (UPM).
\end{abstract}

Palabras clave: tecnología contactless; análisis de datos; control de asistencia; control de acceso

Abstract- Nowadays it is very common that the universities offer to their students an identity card to be used within the facilities of the university as well as out of the university context. It is also more frequent that those cards incorporate the last technologies such as NFC or RFID (contactless technologies) to allow a more dynamic use of the card. This paper explores the development a new service to be implemented in the universities by means of using identity cards with contactless technologies and mobile devices. The goal of the service is twofold: on one hand it aims to control the access of the users to the university libraries; on the other hand it aims to be used in the classroom to control the attendance. Finally, the project has several other exploitations and additional use cases focused on analysing and encouraging the academic performance. The project is under implementation in Universidad Politécnica de Madrid (UPM).

Keywords: contactless technology; data analysis; attendance control; access control

\section{INTRODUCCIÓN}

La digitalización del control de acceso a las dependencias en la universidad tiene el valor añadido de poder analizar los datos y establecer políticas que permitan un mejor uso de los recursos adaptados a los usuarios que lo utilizan.

Dada la naturaleza y el tamaño de las universidades de ámbito presencial es habitual que estén integradas por diferentes campus o localizaciones. Cada uno de los campus, suele contar con diferentes tipos de instalaciones entre las que cabe incluir instalaciones deportivas, bibliotecas, etc. El control de acceso a estas instalaciones se puede realizar de diferentes formas, como es el sistema de tornos, puertas de apertura con código o identificación por carné, control de acceso manual, etc.

En el contexto de los alumnos, es habitual que las universidades ofrezcan algún tipo de carné que les permita ser identificados como estudiantes de dicha universidad, así como darles acceso a sus instalaciones además de otros posibles usos fuera del ámbito estudiantil. La explotación que la universidad pueda hacer de los datos derivados del uso del carné compete directamente a las universidades, aunque para este proyecto se plantean objetivos relacionados con el rendimiento académico principalmente.

\section{CONTEXTO}

En la actualidad las universidades suelen entregar a los estudiantes matriculados en alguno de los estudios que oferta 
un carné universitario. Este carné sirve a los estudiantes dentro de la universidad principalmente como método de identificación para acceder o usar diferentes instalaciones (biblioteca, acceso a aulas informáticas, acceso a laboratorios, etc.).

Las versiones más modernas de este tipo de carné incorporan la tecnología NFC (Near Field Communication). NFC es una tecnología inalámbrica, ampliamente conocida por ser utilizada en entornos de tarjetas de crédito para el pago contactless.

Las tecnologías contactless y el carné que proporcionan las universidades permiten maximizar el potencial del propio carné para que pueda ser utilizado en otros entornos diferentes a los ya descritos tal y como se puede observar en la literatura previa. Ejemplos de este uso incluye el desarrollado por la Universidad Pontificia de Salamanca (Fernández, Fernández, Aguilar, Selvi, \& Crespo, 2013) o el sistema TouchIn (Ayu \& Ahmad, 2014). No obstante, en el contexto del control de asistencia a clase estas no son las únicas iniciativas ya que la prensa se ha hecho eco de otras iniciativas en otros centros como la Universidad de Salamanca (Wayerless, 2012) usando NFC o la Universidad de Malaga mediante un sistema biométrico (Ciencia explicada, 2011). Con una línea en cierto modo continuista respecto a estas iniciativas, en este proyecto se ha diseñado y desarrollado un sistema para la identificación de usuarios (en este caso alumnos). Concretamente, y para plantear ciertas diferencias respecto a enfoques previos, se han planteado dentro del proyecto dos casos de uso que además de permitir mejorar considerablemente el rendimiento del uso que se hace del carné en la actualidad, permitirá en el futuro poder explotar los datos asociados con el objetivo de obtener patrones que, entre otras cosas, sirvan para analizar el rendimiento académico o detectar fraudes en la utilización de los servicios. Todo ello servirá como soporte a los procesos de toma de decisión encaminados a mejorar diferentes procesos en el ámbito universitario.

A continuación, se describen los dos casos de uso, así como las potenciales aplicaciones y mejoras que se consiguen con la solución aportada en este proyecto.

\section{A. Control de acceso a bibliotecas}

El proyecto CARD-CONTROL (Rodríguez-González, A. et al., 2017) plantea como uno de sus objetivos el desarrollo de un sistema que, basándose en el uso del carné de la universidad, permita realizar una monitorización en tiempo real del nivel de ocupación de las diferentes bibliotecas, así como controlar los posibles fraudes que se puedan producir en el uso del carné.

En la actualidad, las diferentes bibliotecas de las universidades controlan el acceso de los estudiantes de diferentes maneras. En el caso de la UPM, cuando hay una gran afluencia de público como ocurre en época de exámenes, los trabajadores de las bibliotecas verifican la identidad de los usuarios a través del carné. Por otra parte, aprovechan dicha verificación para llevar un registro de entradas de usuarios, centrándose en este caso en el almacenamiento de los DNI de los usuarios en un listado, omitiendo otro tipo de información (hora, día de registro).

Por otra parte, la Biblioteca Universitaria de la UPM, actualmente dispone de una página web en la que es posible consultar el estado de ocupación de una sede de biblioteca concreta. Sin embargo, el nivel de ocupación se mide de forma completamente manual por los trabajadores de la biblioteca, dando lugar a que la información no siempre esté actualizada y pueda contener errores.

Es importante resaltar también que, en las épocas de alta demanda de los servicios de la biblioteca, es habitual que las bibliotecas se saturen con largas colas para entrar en las mismas. En estos casos, el acceso a las bibliotecas está restringido a los estudiantes de la propia universidad (normalmente es una biblioteca de acceso público), lo que implica tener que realizar un control de los usuarios que quieren entrar en la biblioteca para verificar que son estudiantes o personal con permiso para usar las instalaciones. Este proceso de verificación, se realiza manualmente por parte del personal de la biblioteca, lo que implica que generalmente este proceso es lento, y además no permite tener en cuenta posibles situaciones de fraude.

Estas situaciones de fraude hacen referencia por ejemplo al hecho de que una persona intente entrar en la biblioteca teniendo un carné a pesar de que haya dejado de ser alumno o personal, o que para esa persona exista más de un carné (por haber solicitado un duplicado por una hipotética pérdida o robo), o situaciones de índole similar.

\section{B. Control de asistencia en clase}

El segundo objetivo de CARD-CONTROL es utilizar las herramientas desarrolladas para poder realizar un control de la asistencia a las clases, utilizando como dispositivo un teléfono móvil y las tarjetas de la universidad.

Con la adaptación de los títulos universitarios al Espacio Europeo de Educación Superior, se empezó a incluir como obligatoria la asistencia a las clases en un gran número de asignaturas. La asistencia obligatoria implica que el docente debe dedicar un tiempo de la clase a realizar el control de esta asistencia. Siguiendo métodos tradicionales la estrategia más habitual era entregar una hoja de firmas a los alumnos para asegurar su presencia en el aula. En su versión más optimizada, esta hoja de firmas ya contenía el nombre y apellidos del alumno y estaban ordenados para que simplemente firmaran. En la peor, la hoja estaba en blanco y el alumno debía escribir su nombre y firmar. En una asignatura de 6 ECTS (European Credit Transfer and Accumulation System) donde se dan $4 \mathrm{~h}$ de clase a la semana repartida generalmente en 2 clases de $2 \mathrm{~h}$, durante todo el periodo del cuatrimestre, esto implica que durante las aproximadamente 15 semanas del cuatrimestre, el docente debe pasar unas 30 hojas de firmas por cada una de las asignaturas o grupos que imparta. En asignaturas con gran número de alumnos (por ejemplo 280), donde se suelen dividir en 3 grupos por las capacidades de las aulas, esto implica tener unas 90 hojas de firmas a lo largo del cuatrimestre.

Cuando el docente debe realizar el computo de qué alumnos han asistido a las clases (para otorgar un valor por asistencia en la evaluación continua o el criterio de evaluación que implique la asistencia), esto supone la revisión de 90 hojas de firmas, con el fin de realizar una distribución de cuáles de los 280 alumnos han asistido a las 30 clases que se han impartido durante todo el cuatrimestre. En el caso de disponer de hojas de firmas donde ya estaban los nombres de los alumnos impresos de forma alfabética, esta tarea, si bien conlleva tiempo, es factible. Si por el contrario las hojas entregadas 
eran hojas en blanco y el docente tiene que identificar el nombre que ha escrito el alumno y además no sigue ningún orden en la hoja, esta tarea se convierte en una tarea muy tediosa. En el primero de los casos (el más sencillo y fácil de evaluar), podemos estimar que el tiempo aproximado medio en pasar estos datos a un entorno computarizado (una hoja de cálculo) es de unos 5s por alumno. El paso a la hoja de cálculo de todos los alumnos (280) de un solo día implica la friolera de 1.400 segundos (unos 23 minutos). Con 30 partes de hojas de firmas esto implica cerca de $12 \mathrm{~h}$ de trabajo en el mejor de los casos, partiendo nuevamente de que esa media de 5 segundos no se viera incrementada por factores como el cansancio. Por otra parte, la probabilidad de que se produzca algún error a la hora de transcribir los datos es extremadamente alta dada la naturaleza de los datos y las herramientas a utilizar.

Si extrapolamos este esfuerzo en tiempo al número total de asignaturas y docentes que controlen la asistencia en un centro docente, el número de horas que se puede perder en esta tarea, por parte de los diferentes profesores de dicho centro, es enorme. Tiempo que el docente podría invertir en otras tareas mucho más importantes relacionadas con su docencia, investigación o gestión.

\section{DESCRIPCIÓN}

El proyecto CARD-CONTROL se enmarca en un Proyecto de Innovación Educativa (PIE) financiado por la Universidad Politécnica de Madrid. Los objetivos ya descritos se traducen en dos tareas:

1. Analizar y escoger la mejor herramienta o dispositivo a nivel hardware que permita implementar el software a utilizar para cumplir con los objetivos descritos en el proyecto

2. Diseño y desarrollo de un software que permita la lectura de los carné de la universidad a través de un dispositivo móvil, así como los posibles servicios intermedios para dar la funcionalidad requerida a los casos de uso de acceso a la biblioteca y control de asistencias.

La primera de las tareas implica un análisis pormenorizado de los diferentes tipos de dispositivos móviles (teléfonos móviles inteligentes, tabletas) que mediante una relación coste-eficiencia permitan ejecutar el software que se encargará de realizar la lectura de los datos del carné de la universidad. Se estudiaron inicialmente opciones de desarrollo de hardware básico tipo RaspberryPi o Arduino. El investigador principal del proyecto había dirigido un Trabajo Fin de Grado (TFG) donde se desarrollaba un dispositivo basado en estas tecnologías (concretamente Raspberry $\mathrm{Pi}$ ) para crear un dispositivo hardware de bajo coste con el objetivo de controlar la asistencia a clase. Aunque los resultados con el dispositivo piloto creado fueron satisfactorios, se identificaron diferentes problemas de cara a desarrollar este proyecto con este tipo de infraestructuras, fundamentalmente relacionados con el coste final, fragilidad del dispositivo desarrollado y problemas asociados al desarrollo del software al tener que lidiar con componentes hardware muy específicos y no existir una buena abstracción a la hora de desarrollar el código.

Debido a esta identificación de problemas, se decidió optar por plataformas tipo móvil. Aunque en el mercado existen diferentes dispositivos que cumplían con los requisitos básicos (sistema basado en Android con capacidad de lectura de tarjetas NFC y RFID), finalmente se decidió optar por un dispositivo de la empresa FAMOCO, concretamente por el modelo FX-300 (FAMOCO, 2017). El motivo fundamental se basaba en la lectura de códigos de barras. Aunque cualquier móvil con cámara es capaz de leer un código de barras, la lectura se fundamenta en técnicas de reconocimiento de imagen. Esto implica que es necesario que la cámara enfoque correctamente el código para poder leerlo, algo que puede llegar a ser relativamente lento. Sin embargo, el dispositivo FX-300 de FAMOCO tiene directamente un lector laser de código de barras incorporado, lo que facilita la lectura. Además, los dispositivos de FAMOCO están específicamente diseñados para ser utilizados en contextos como el del proyecto, lo cual permite una solución con un coste no muy alto que se adapta bastante a los objetivos (sobre todo al de control de acceso a la biblioteca).

Cada uno de los casos de uso requiere de una arquitectura específica dada las características del caso de uso. A continuación se describen las arquitecturas empleadas para cada uno de los casos y sus principales componentes tecnológicos.

\section{A. Control de acceso en bibliotecas}

El control de acceso a la Biblioteca de la UPM se realiza usando el carné de la universidad. Cuando un alumno o profesor quiera acceder, debe presentar su carné de la universidad al personal de la biblioteca, el cual se encarga de verificar que tiene permitido el acceso leyendo la información del carné mediante el dispositivo FAMOCO FX-300 ya especificado.

Cuando se realiza la lectura del carné, el dispositivo se conecta con una plataforma de servicios central (PSC). El PSC es el encargado de verificar si el usuario tiene permiso de acceso, y en caso afirmativo, si ya está dentro de la biblioteca o no (para registrar entrada o salida) y si hay un posible caso de fraude, al detectar múltiples entradas en un lapso de tiempo en una o varias sedes de la biblioteca o situaciones análogas. En el caso de entrada, el PSC realiza una petición al Servicio de Acceso de Usuarios (SAU), que es un servicio desplegado en los Servicios Informáticos de la Universidad (SIU) y que en base al código leído de la tarjeta NFC/RFID devuelve el DNI del usuario al que pertenece y si tiene permitido el acceso a la Biblioteca en base a los criterios definidos (pago de matrícula; situación en activo; etc.).

La arquitectura global de la plataforma de control de acceso a bibliotecas es la que se refleja en la Figura 1.

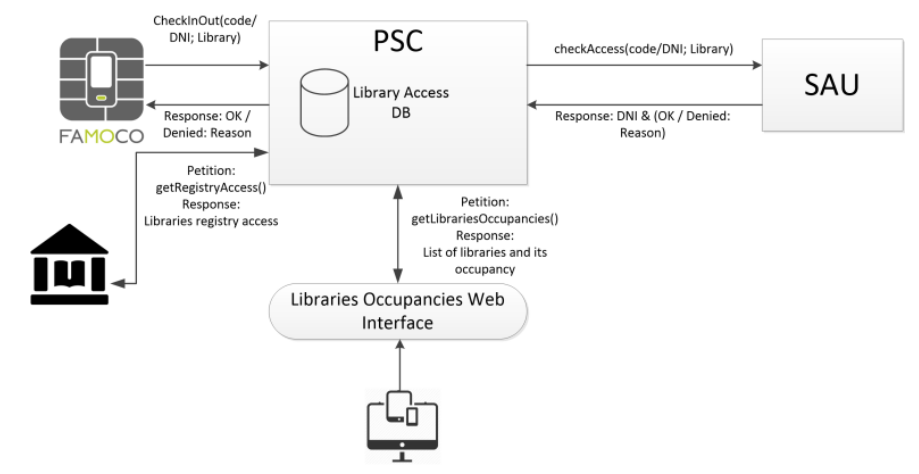

Figura 1. Arquitectura caso de uso de la biblioteca 
A continuación se describe el flujo de información desde los diferentes elementos:

\section{FAMOCO-PSC-SAU:}

El dispositivo FAMOCO tiene instalada una aplicación Android que lee la información del carné de la universidad. Esta aplicación tiene un login inicial para las diferentes sedes de la Biblioteca (ver Figura 2) para enviar los datos de forma correcta. Cada sede tiene unas credenciales de acceso únicas.

La información que lee el dispositivo FAMOCO del carné de la universidad del alumno que quiere entrar es la siguiente: un código único (a través de NFC o RFID (de ahora en adelante Tag)) o el DNI (a través de código de barras (CB) o introducción manual) (ver Figura 3). La aplicación se conecta al servicio PSC (CheckIn o CheckOut) y envía los siguientes datos:

\section{- Código o DNI}

- $\quad$ Sede de la Biblioteca donde se realizó la lectura

- ID del dispositivo FAMOCO

- $\quad$ Método de lectura (Tag, CB, Manual)

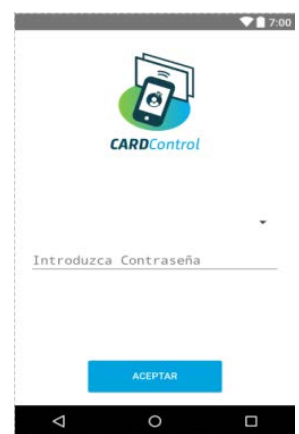

Figura 2. Captura de pantalla del login de CARD-Control Biblioteca

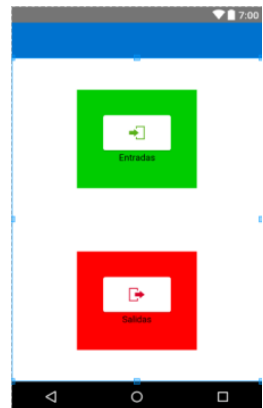

Figura 3. Captura de pantalla de lectura en FAMOCO

El servicio PSC realiza las verificaciones pertinentes relativas a existencia previa de registro de entrada o salida. En caso de ser un proceso de salida (CheckOut) se comprueba en la Base de datos (BD) y se devuelve la respuesta al dispositivo FAMOCO directamente sin conectar al servicio SAU (salvo que haya disparidad de datos en los registros de entradasalida). De cara al proceso de entrada (CheckIn), el PSC realiza en primer lugar una serie de verificaciones relativas a un posible intento de entrada por parte de este usuario en otra sede de la Biblioteca sin haber realizado registro de salida o situaciones análogas que puedan suponer un teórico fraude. En caso de no encontrar fraude, el servicio PSC contacta con el servicio SAU para verificar si el usuario que intenta entrar en la biblioteca tiene permiso para ello (checkAccess). El servicio PSC envía al servicio SAU una información similar a la que envió FAMOCO al servicio PSC:

- Código o DNI

- $\quad$ Método de lectura (Tag, CB, Manual)

- Sede de la Biblioteca donde se realizó la lectura

El servicio SAU es el encargado de consultar las bases de datos de la universidad que sean necesarias para comprobar todas las posibles situaciones que puedan aceptar o denegar una entrada a cualquiera de las sedes de la Biblioteca de la Universidad (existe el usuario; está al corriente de pago de matrícula; no tiene inhabilitado el acceso por alguna razón; etc.). Este servicio devuelve al servicio PSC la siguiente información:

- Código o DNI recibido en la petición.

- DNI del usuario: Si se recibió un código este DNI se obtiene del mapeo de los ficheros de la entidad bancaria. Si se recibió un DNI se devuelve el mismo.

- Respuesta: OK o denegación. En caso de denegación se debe devolver el motivo de denegación (con un código único) y un texto explicativo.

Una vez el servicio PSC reciba esta información la almacena en su base de datos. En caso de denegación de entrada, se guarda en una tabla específica de denegaciones donde se almacena el código, DNI, timestamp, biblioteca, motivo de denegación (código) y texto de denegación. En caso de permitir el acceso, se guarda en otra tabla diferente los siguientes datos: código, DNI, biblioteca, método de entrada, dispositivo FAMOCO y hora de entrada. A continuación devuelve la respuesta al dispositivo FAMOCO.

\section{BIBLIOTECA-PSC:}

Los servicios centrales de la Biblioteca Universitaria tienen acceso al servicio PSC para poder descargar los datos de acceso a las diferentes bibliotecas. Para ello puede realizar una llamada al servicio PSC (getRegistryAccess) y obtener los registros de entrada de una o varias bibliotecas proporcionando diferentes parámetros (tiempo inicio y tiempo final; biblioteca específica, etc.). El servicio devuelve el registro de acceso en un formato que la Biblioteca Universitaria pueda consultar y cargar para analizar (por ejemplo: CSV).

\section{USUARIO-PSC:}

Los usuarios finales (alumnos, personal,...) pueden realizar consultas al servicio PSC a través de una interfaz web. Esta interfaz permite mostrar los niveles de ocupación de las bibliotecas en tiempo real en función de los registros de entrada y salida almacenados. La interfaz web llama al servicio que devuelve esta ocupación 
(getLibrariesOccupancies) para visualizar la información devuelta. La Figura 4 muestra una representación de lo que ven los usuarios, en este caso en un ordenador normal.

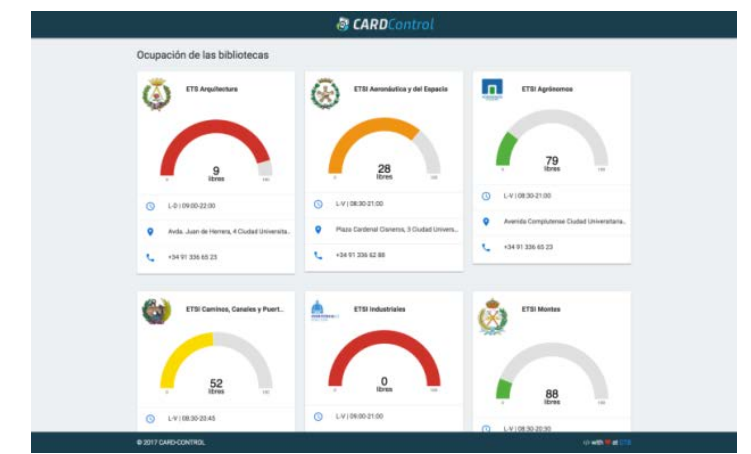

Figura 4. Captura de pantalla de los datos de ocupación

\section{B. Control de asistencia}

El control de asistencia se realiza mediante una configuración inicial que realiza el profesor que desea controlar la asistencia de una determinada asignatura en un determinado horario. El profesor utiliza el dispositivo móvil (en este caso no necesariamente tiene que ser el dispositivo FAMOCO; se puede usar un móvil que permita leer NFC/RFID con cámara para la lectura del código de barras, ya que no es necesaria la lectura por láser, más veloz, que sí se requiere en el caso de uso de control de acceso a las bibliotecas). Una vez el profesor configura el dispositivo y selecciona la clase de la que va a controlar la asistencia (asignatura, fecha, grupo, etc.), entrega el dispositivo a los alumnos para registrar los datos y se devuelve al profesor. La arquitectura de esta plataforma se puede ver reflejada en la Figura 5.

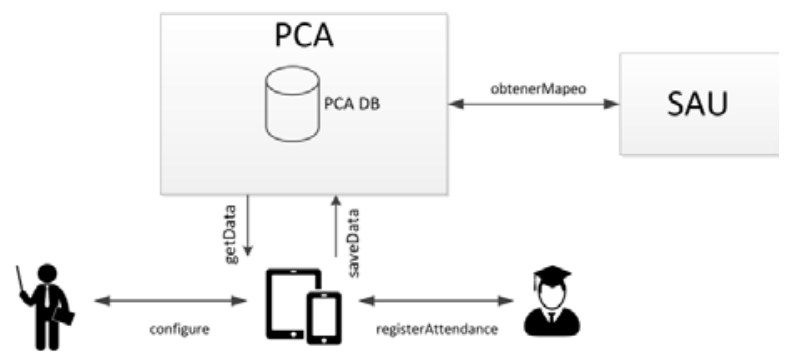

Figura 5. Arquitectura caso de uso de control de asistencia

Una vez en mano de los alumnos, el dispositivo muestra detalles sobre la asignatura y la fecha en la que se está controlando la asistencia y dos botones (control manual y finalizar) (ver Figura 6) que en caso de ser pulsados pedirán un código que deberá introducir el profesor. El botón de control manual permite hacer una lectura de carné a través del código de barras. Solo se permite que esta lectura se realice frente al profesor ya que el código de barras es un sistema muy fácilmente falsificable, con lo que el control por este medio sería manual. Al pulsar el botón de finalizar, finaliza la lectura de los códigos y hace que el dispositivo se conecte con la Plataforma de Control de Asistencia (PCA).

La PCA recibe del dispositivo móvil la siguiente información: asignatura a controlar, profesor, horario de control y el listado de códigos de carné leídos. La PCA se conecta con el servicio SAU para enviarle los códigos de los carné. En este caso y a diferencia del caso de la biblioteca, el SAU simplemente devuelve para cada ID de carné leído, cual es el DNI y el número de matrícula asociado, pero no realiza comprobaciones adicionales (como si está al corriente de pago de la matrícula). La información se devuelve a la PCA, que la almacena. La PCA realmente realiza, antes de conectar con el SAU, una comprobación de IDs. Cuando un alumno atiende a una clase por primera vez y se pide al SAU sus datos a través del ID de su carné estos datos se almacenan, y se verifica siempre si ya se tienen para evitar enviar al SAU más datos de los necesarios. Esto además permite detectar fraudes. Supongamos que un alumno dispone de dos carné (C1 y C2) porque pudo obtener una copia ya que dijo que había perdido uno de ellos. Tiene que ir a dos asignaturas que son a la misma hora y en las que se controla la asistencia, pero como no tiene el don de la ubicuidad decide ir a la clase de Bases de datos de 10 a 12 y pasar ante el control de asistencia el carné $\mathrm{C} 1$, y le deja a su compañero el carné C2 para que lo pase por la clase de Concurrencia que es en el mismo horario. El sistema detectará que se están haciendo uso de dos carnés para un mismo usuario, y además podrá detectar el solapamiento de las asignaturas donde se ha controlado la asistencia.

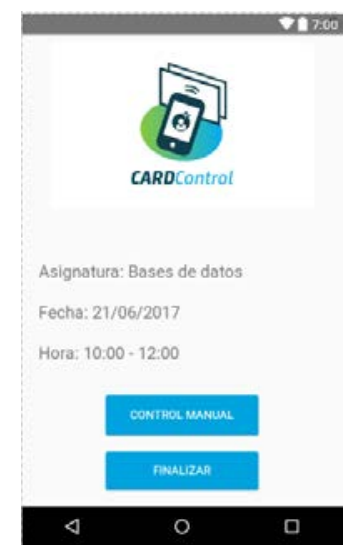

Figura 6. Captura de pantalla del proceso de control de asistencia

La conexión con el SAU se realiza solamente cuando el profesor indica que ha finalizado el proceso de control de la asistencia y envía todos los datos de una vez para evitar saturaciones del servicio y múltiples y repetidas llamadas.

La información se almacena en la base de datos, de tal forma que cuando acabe el curso, un profesor puede obtener estadísticas detalladas de la asistencia a su clase (alumno que más ha asistido, medias de asistencia, que alumnos han asistido a un porcentaje mínimo, etc.).

\section{RESUltados}

El proyecto CARD-Control está en su fase final de desarrollo y de prueba para su implantación final. El caso de uso de la biblioteca se aplicará una vez estén disponibles las herramientas finales, con una primera fase, en la que solo aquellas bibliotecas que UPM estime oportuno, formarán parte del proyecto piloto.

En lo que se refiere al control de asistencia de alumnos a las clases, se implementará un primer piloto durante el curso académico 2017-2018 en la asignatura Bases de datos de la Escuela Técnica Superior de Ingenieros Informáticos de la Universidad Politécnica de Madrid. 


\section{CONCLUSIONES}

La solución propuesta tiene como objetivo mejorar los sistemas de control a las dependencias universitarias. En primer lugar, mediante un uso más eficiente del carné que la universidad proporciona a todo estudiante. A pesar de que el carné ha ido incorporando las tecnologías más recientes, nunca se ha hecho un uso directo del mismo, de tal forma que la universidad, y la comunidad universitaria en general, pudieran beneficiarse. Por lo tanto, este proyecto supone un primer uso en un contexto y entornos reales. Además, el control de accesos digitalizado a la biblioteca y a las aulas permite almacenar información de los accesos, datos que podrán ser posteriormente analizados como soporte a procesos de toma de decisión. En el caso del acceso a la biblioteca, este análisis de datos podría permitir mejorar los procesos internos de la misma en lo que se refiere al control de acceso y la continua actualización de los puestos disponibles. Pero además, permitirá a la Biblioteca Universitaria disponer de información de gran valor que propiciarán el análisis en detalle en función de los niveles de ocupación de los servicios que ofrecen y como mejorarlos. En cuanto al control de asistencia, como ya se ha comentado previamente, el tiempo que dedica un docente de media a las tareas relacionadas con el simple hecho de cotejar firmas para ver que alumnos han asistido a que clases es increíblemente alto. El uso de esta plataforma permitirá automatizar estas tareas y permitirá que ese tiempo pueda ser empleado de otras actividades. Finalmente, para ambos casos los datos podrán además ser analizados y obtener patrones de asistencia a clase dependiendo de distintos factores (fechas, horarios, cercanía a los periodos de exámenes,...) que podrán ser usados para mejorar los procesos educativos y estudiar la correlación existente entre la asistencia a clase y el rendimiento académico tal y como se ha hecho previamente (Andrietti, 2014; Andrietti \& Velasco, 2015; Grabe \& Christopherson, 2008).

\section{LINEAS FUTURAS}

El desarrollo del proyecto deja abiertas varias líneas futuras, relacionadas con la explotación de los datos a adquirir. En primer lugar debe destacarse el análisis de los datos para estudiar posibles mejoras del servicio que ofrece. Con los datos que se podrán obtener, la Biblioteca podrá hacer una mejor reorganización del personal en función de las horas o momentos de mayor afluencia, podrá gestionar mejor los espacios y en general podrá mejorar y optimizar sus servicios y recursos. Además, estos datos pueden ser muy importantes para realizar análisis cruzado con otras fuentes, como podrían ser por ejemplo las relacionadas con rendimientos académicos para estudiar las posibles correlaciones entre el uso de la biblioteca y un mejor o peor rendimiento académico.

De igual forma, en el caso de uso del control de asistencia a clase, existen muchas posibilidades en lo que se refiere al análisis de los datos. En primer lugar se puede analizar si las aulas destinadas a la impartición -de ciertas asignaturas cubren la demanda. También se puede ver la evolución de la asistencia a clase y cotejarlo con posibles solapamientos con otras asignaturas o con el rendimiento académico. Se podrá estudiar si el rendimiento académico de un alumno decae cuando deja de ir a clase y dar respuesta a muchas más preguntas que sin duda podrían mejorar los procesos educativos. Finalmente, y aunque este proyecto no lo contempla, una línea futura podría incluir el aprovechar la infraestructura montada para reducir la carga de trabajo asociada a los coordinadores de las asignaturas en las fases de volcado de horas impartidas, ya que el propio dispositivo podría registrar la distribución de horas impartidas por cada profesor y enviarlas a los departamentos oportunos de forma directa.

\section{AgRAdECIMIENTOS}

Esta publicación ha sido financiada gracias al Proyecto de Innovación Educativa con código IE1617.1002 de la Universidad Politécnica de Madrid.

\section{REFERENCIAS}

Andrietti, V. (2014). Does lecture attendance affect academic performance? Panel data evidence for introductory macroeconomics. International Review of Economics Education, 15, 1-16. https://doi.org/10.1016/j.iree.2013.10.010

Andrietti, V., \& Velasco, C. (2015). Lecture Attendance, Study Time, and Academic Performance: A Panel Data Study. The Journal of Economic Education, 46(3), 239259. https://doi.org/10.1080/00220485.2015.1040182

Ayu, M. A., \& Ahmad, B. I. (2014). TouchIn: An NFC Supported Attendance System in a University Environment. International Journal of Information and Education Technology; Singapore, 4(5), 448-453. https://doi.org/http://dx.doi.org/10.7763/IJIET.2014.V4. 448

Ciencia explicada. (2011). La Universidad de Málaga implantará un sistema de control de asistencia mediante huellas dactilares único en el mundo. Retrieved July 11, 2017, from http:/www.cienciaexplicada.com/2011/03/la-universidad-de-malagaimplantara-un.html

FAMOCO. (2017). FAMOCO. Dispositivo FX-300. Retrieved from https://www.famoco.com

Fernández, M. J. L., Fernández, J. G., Aguilar, S. R., Selvi, B. S., \& Crespo, R. G. (2013). Control of attendance applied in higher education through mobile NFC technologies. Expert Systems with Applications, 40(11), 4478-4489. https://doi.org/10.1016/j.eswa.2013.01.041

Grabe, M., \& Christopherson, K. (2008). Optional student use of online lecture resources: resource preferences, performance and lecture attendance. Journal of Computer Assisted Learning, 24(1), 1-10. https://doi.org/10.1111/j.1365-2729.2007.00228.x

Rodríguez-González, A. et al. (2017). CARD-CONTROL: Uso del carné universitario para el control de alumnos y los usos de las instalaciones en la universidad. Retrieved July 11, 2017, from http://innovacioneducativa.upm.es/proyectosIE/informac ion?anyo=2016-2017\&id=2226

Wayerless. (2012, January 30). España: Tecnología NFC para controlar la asistencia en la Universidad de Salamanca. Retrieved July 11, 2017, from https://www.wayerless.com/2012/01/espana-tecnologianfc-para-controlar-la-asistencia-en-la-universidad-desalamanca/ 